\title{
Correction to: Evaluating the impact of topological protein features on the negative examples selection
}

Paolo Boldi ${ }^{\dagger}$, Marco Frasca ${ }^{*}$ and Dario Malchiodi ${ }^{\dagger}$

Correction to: BMC Bioinformatics 2018, 19(Suppl 14):417

https://doi.org/10.1186/s12859-018-2385-x

After publication of the original article [1], it was noticed that the dagger symbol indicating equal contribution wasn't added next to the names of all authors. The author list in this correction article has been modified to represent this correctly.

Published online: 17 December 2018

\section{Reference}

1. Boldi P, Frasca M, Malchiodi D. Evaluating the impact of topological protein features on the negative examples selection. BMC Bioinf. 2018;19(Suppl 14): 417. https://doi.org/10.1186/s12859-018-2385-x.

\footnotetext{
* Correspondence: frasca@di.unimi.it

${ }^{\dagger}$ Paolo Boldi, Marco Frasca and Dario Malchiodi contributed equally to this work. Department of Computer Science, Università degli Studi di Milano, Via Comelico 39, 20135 Milano, Italy
} 